\title{
A Psychology Expert System to Determine the Level of Stress in Subjects
}

\author{
Ali Mirzapour
}

\begin{abstract}
As programs that imitate an expert's behavior in a specific area, expert systems can be used, trusted and influential in different areas due to the modeling of the human's logic and reasoning system, and similarity of the sources of knowledge used by them. In the absence of experts, the intelligent software can measure the level of stress in individuals to a relatively reliable level. The design of intelligent systems in psychological counseling is of great importance due to the impact of this field on the various areas of today's life. The present paper aims to describe how to design and implement a psychology expert system to determine the level of stress in different people using MATLAB software. As one of the most popular psychological tests to determine the level of anxiety, depression and stress in different individual, this test is conducted based on the DASS-21 test, which deduct the result depending on the rules defined and the responses received from the user and determines the level of stress of the individual.
\end{abstract}

Index Terms-Psychology Expert System, Stress Assessment, level of stress, MATLAB software

\section{INTRODUCTION}

As stress can cause many diseases, including heart problems, headaches, migraines and sleep problems, diagnosing stress is of great important. On the other hand, the design of intelligent systems in the psychological counseling is very vital due to the impact of this field on different areas of life today.

Expert systems are designed with the goal of making expert skills available for non-expert individuals. These programs simulate the human's pattern of thinking and performance and close the performance of expert systems to that of a person or an expert.

In fact, an expert system is a computer system that simulates an expert's decision-making ability. The main idea of the expert systems is raising the benefits achieved by individuals of expert knowledge. Such systems include inbuilt capabilities to write rules that create the knowledge base [1]. Also, a computational technique like Data Envelopment Analysis for other health care issues such as heart disease has been reported in the literature [2].

Expert system significantly comforts clinical effort by helping scientists to take the best medical decision at the foundation of the young patient's treatment [3].

The sympathetic nervous system plays a role in stress situations with all aspects of increased secretion of adrenaline. The device measures the energy level of the entire body shown by Energy parameter which is the average of energy standards determined in 24 energy points situated on the skin external of hands and feet, corresponding to several organs and systems. It also assesses the stress standing of the autonomic nervous system connected with signs and gives us suggestions of possible dangers for the body health when these values are enlarged [4].

An expert medical system is a computer program. If they are applied appropriately, they help in effective diagnosis of diseases and their treatment such as nephrolithiasis [5] and liver diseases [6].

\section{EXPERT SYSTEM}

Decision-making systems, or generally, computer-based decision making, have become increasingly important by expanding the application of information technology. In this regard, as one of the branches attributed to artificial intelligence, expert systems have a major role. A variety of decisions can be made by computers in expert systems, as knowledge-based systems with knowledge as their most important part [7]. Hence, the quality of the expert system depends on its knowledge base. In expert systems, which are widely used in various sciences in the world, knowledge is transferred from the experts of every field of science to the computer. Among the areas of designing and presenting these systems are industry, control, astronomy, financial decision making, etc. Due to factors such as budget deficits, turnover, and so on, the use of expert medical systems has been considered [8].

Some of the advantages of expert software systems include the following:

- The individuals' expertise, unlike computer expertise, is temporary and mortal. For example, a person may change his job, become sick, and so on.

- Individual expertise is not stable. Holidays, recreational programs, etc. all can affect the natural person's performance, but the computers are stable and provide the same outputs in all conditions.

- The individuals' expertise is hard to transfer. One person cannot be present at two locations at one time, but computer expertise is transferable. For example, an expert system installed on a computer is able to copy to another computer elsewhere, or even can be downloaded through the network.

- The individual's expertise is usually expensive. Personnel salaries are much higher than the cost of hardware and software.

- Expert systems have the ability to upgrade.

There are other benefits of expert systems including higher efficiency, full and fast response, good reliability, comprehensibility, flexibility, lower risk, durability and survival, and multiple expertise [9]. This computational method is obvious in other fields such as Data Envelopment Analysis(DEA) in operation research and data mining [10]. 
There are four main parts for an expert system model:

1. Knowledge Base

2. Inference Engine

3. Explanation Facilities

4. User Interface

\section{A. Knowledge Base}

A place where expert knowledge is coded and become understandable for the system. The knowledge base can be considered as a list of objects where the rules and indexes of each object are also mentioned.

\section{B. Inference Engine}

As the heart of an expert system, the inference engine is actually a complex system of the inference rules that uses a set of "if ... then ...." rules to find the answer or the final judgment. What turns this system into an expert system is the way in which these rules are processed. The inference system can be applied in two ways in order to achieve a judgment, and in fact, pass through the hierarchy of rules of reasoning, in two ways. One of the two methods is the forward reasoning method that starts from the data and come to the conclusion, that is, it starts with considering the data of the subject with (ifs) questions and achieve the appropriate results or (thens); in other words, we get results from the basics. The second method is deduction method which starts from results and seek appropriate basics of primary conditions for that specific results. In other words, the starting point is (thens) and (ifs) are achieved from them. The first method of deduction is based on the data method and the second method is based on the objective method.

\section{Explanation Facilities}

This component is used to illustrate the conclusion stages of an expert system for a specific problem with specific facts and using an understandable language for the user.

\section{User Interface}

The user interface refers to a set of hardware and software that acts as a channel for user and expert communication, which allows the user to provide information about the problem to the system and, on the other hand, provides the system's inferences to the user. For the purpose that the information exchange structure takes the form of an interview between an applicant and an expert person, the user interface of an expert system should naturally have a high exchange power.

\section{THE SUBJECT IMPORTANCE AND NECESSITY}

One of the problems of psychological counseling is that individuals avoid giving the correct answer because they do not want to introduce themselves as patient, which also causes the counselor to not be able to correctly identify their problem, and finally, the patient will not achieve the desired outcome that should be obtained from the consultation session.

By designing the expert system, it is possible for an individual to respond to questions without observing many issues and, as a result, recognize their real level of stress, and then seek a suitable solution to deal with this problem.

The proposed expert system is designed on the basis of a DASS-21 psychological test (test is provided in appendix) which is one of the most widely used tests to measure stress, anxiety and depression in different individuals. Due to the complexity of the project, the two factors of anxiety and depression has been ignored and only the stress factor has been studied. Hence, questions 1, 6, 8, 11, 12, 14, 18 of the stress test for DASS-21 are asked and the user is obliged to answer the questions with high accuracy. The other two can be similarly added by adding the related questions to the corresponding knowledge base.

\section{The SubJeCt THEORY}

Firstly, the rules of the expert system are defined and stored in the database. Next, the questions about stress measurement are appropriately answered by individuals through the user interface screen, and the results are stored in a database. Then, an inference engine performs the inference based on the rules defined above and the individual's responses. Finally, the outputs of the expert system are displayed which represent the degree of stress in each patient.

\section{THE EXPERT SYSTEM IMPLEMENTATION}

The proposed expert system implementation can be done by any of the programming languages that in this article, it is performed using MATLAB software.

The stress assessment questions are firstly asked by the expert system from the user or patient in the Input Box. The user is obliged to choose the correct answers for the following questions:

1. Do you extremely react to the situations? [0 100]

2. How difficult is to keep yourself calm? [0 50]

3. How impatient are you to anything that hamper your work? [0 100]

4. How irritable are you? [0 100]

Each of the questions raised by the expert system is considered as an object that various answers can be given by the user as the indicators. Also, the shape of the associated function fitted it is designed according to the stored information. Given the questions and possible answers, the membership function is considered as triangular.

\section{A. Question No. 1: Do you react extremely to situations? (The answer should be a value between 0 and 100)}

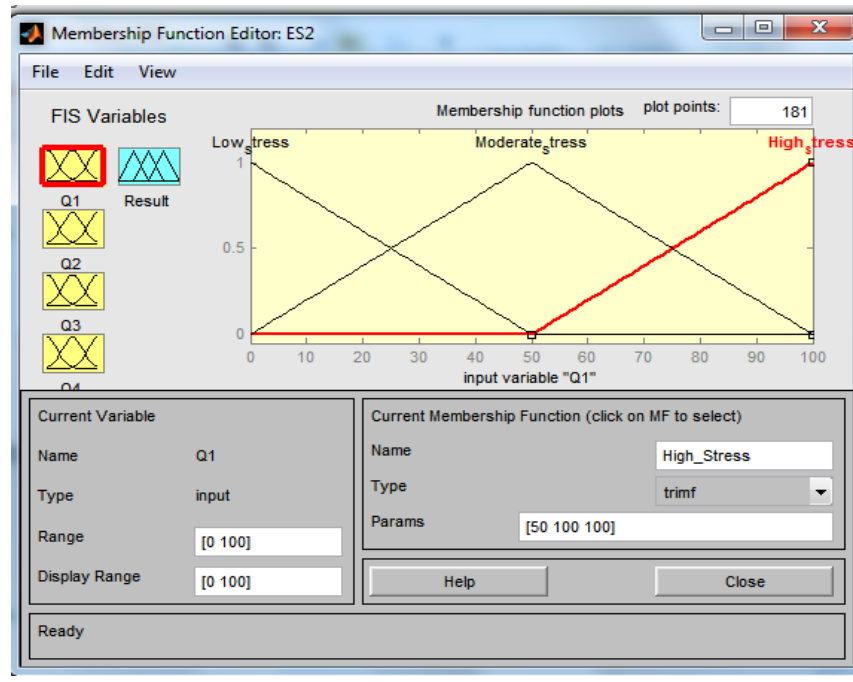

Fig. 1. Membership function of the first question 
B. Question No. 2: How difficult is for you to calm down and relax? (The answer should be a value between 0 and 50)

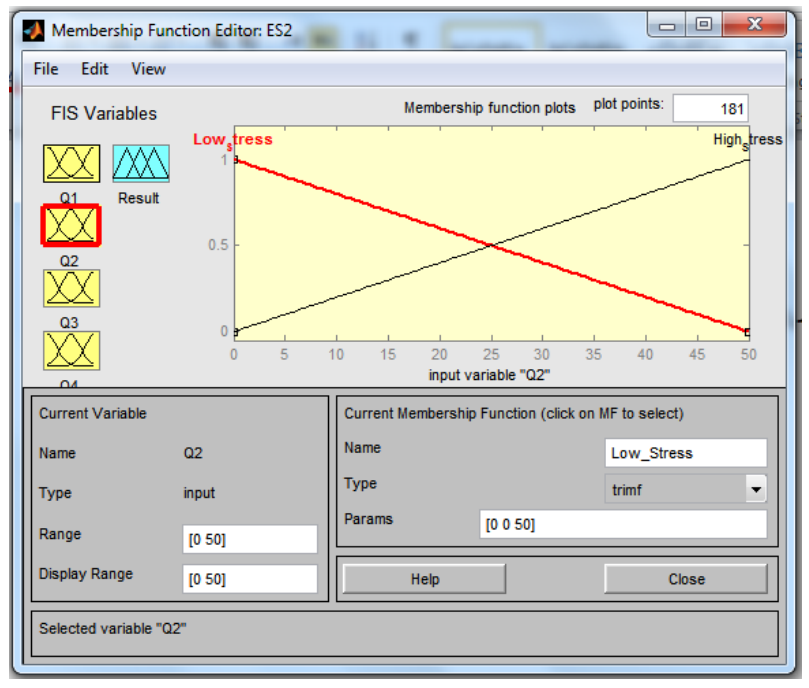

Fig. 2. Membership function of the second question

\section{Question No. 3: How much intolerant and impatient} you against things that hinder you? (The answer should be a value between 0 and 100)

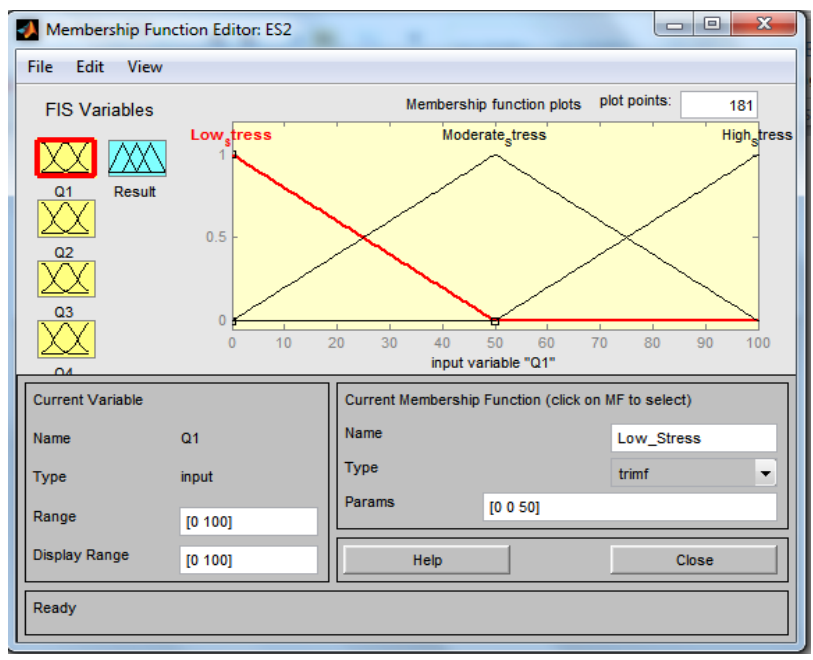

Fig. 3. Membership function of the third question

D. Question No. 4: How much sensitive and irritable you are? (The answer should be a value between 0 and 100)

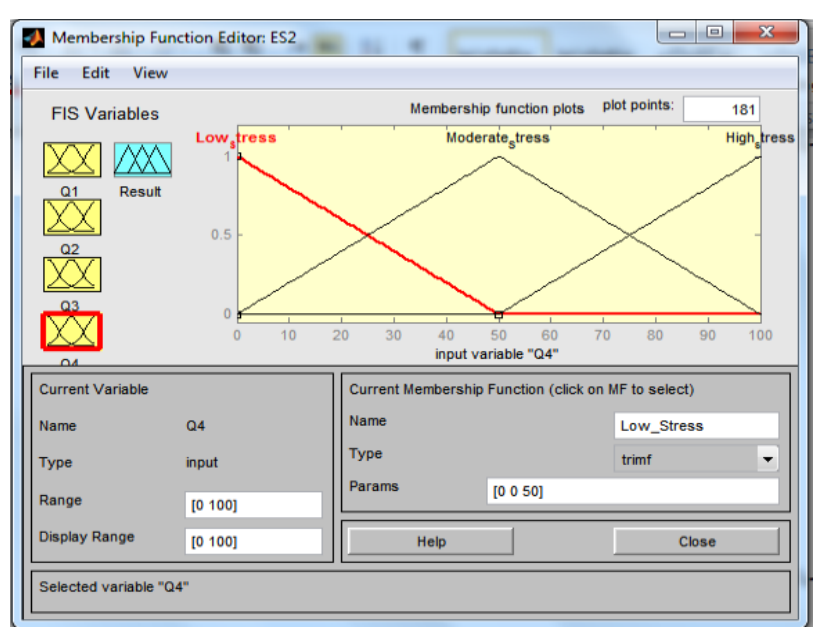

Fig. 4. Membership function of the forth question

\section{E. Determining the rules}

The relevant rules for the expert system are determined at this stage that will be used by the inference engine in the next step.

\section{F. Designing the inference engine}

The expert system performs the inference operations in accordance with the rules and the type of combination considered from the set of possible modes.

\section{G. Rule viewer}

Fig.5. shows the rule viewer.

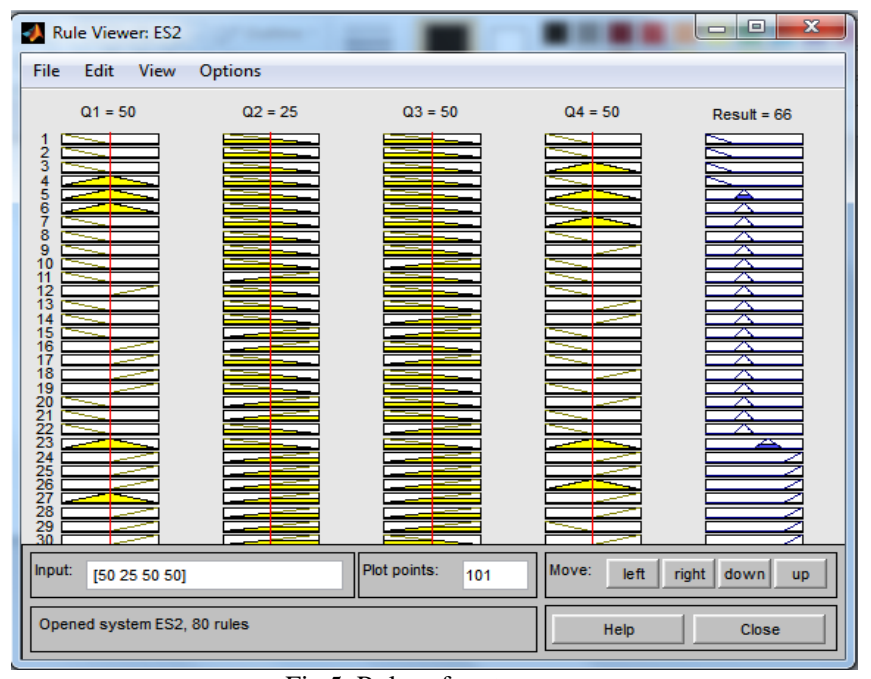

Fig.5. Rules of system

\section{H. Surface view}

Surface view of input and output is presented in Fig. 6.

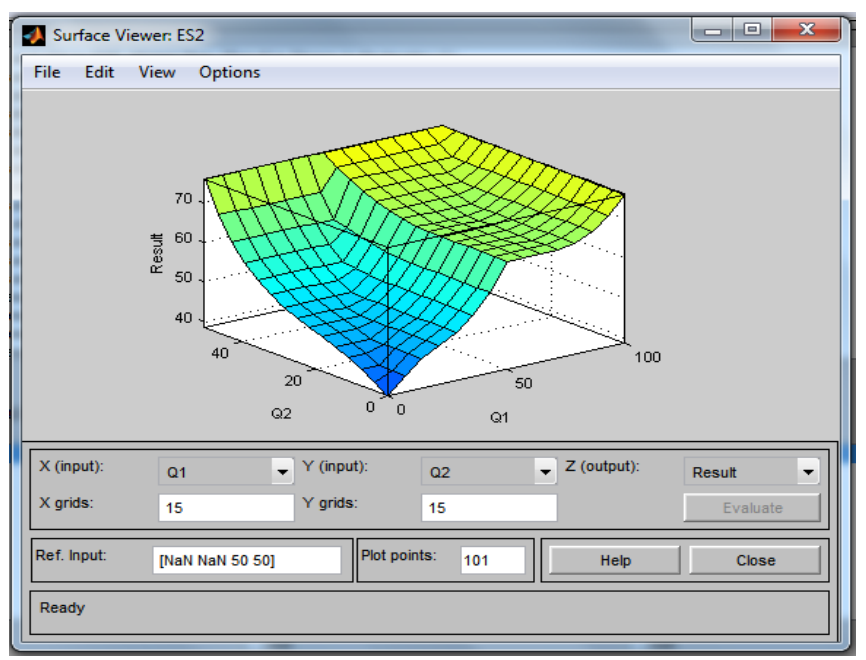

Fig.6. Surface view of input and output

\section{Outputs}

The designed expert system should declare the patient's stress according to the patient's responses and the rules defined by the expert. The output is presented as one of the following forms:

- $\quad$ Output 1: user is normal, that is, the person has no signs of stress.

- $\quad$ Output 2: user has a mild level of stress. 
- Output 3: user has a moderate level of stress.

- Output 4: user has a high level of stress.

If the value obtained from the above numerical relationship is between 0 and 30, the output 1 will be declared to the user; if it is between 30 and 50, the output 2 will be declared; if it is between 50 and 80 , the output 3 will be declared and finally, it is between 80 and 100, the output 4 will be declared to the user. The output diagram is available in Fig. 5.

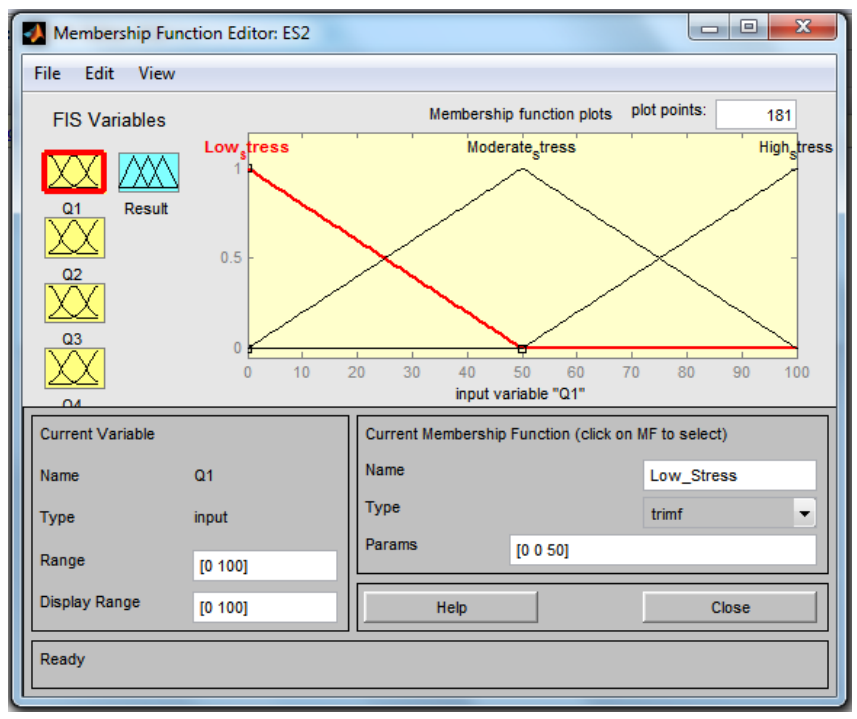

Fig.7. Output diagram

\section{CONCLUSION}

In this paper, a psychology expert system is designed to show the degree of stress in individuals. The function of this system is in the form that it initially poses questions and, enters the operational stages after receiving the response from the user. In this way, it begins to deduce and announce the result to the user based on the responses received from the user and the defined rules. The intelligent and semi-intelligent systems such as expert systems can be used as a decision support system. However, there is still a long way to completely substitute these systems with the experts. The use of intelligent and semi-intelligent systems such as expert systems can be made available to decision makers. However, at present, it cannot replace the experts.

\section{REFERENCES}

[1] A.V. Saurkar., and H.N. Watane. An expert system for diseases diagnosis in pet, vol.2, 1, pp.18-21, 2012.

[2] M. Mirmozaffari and A. Alinezhad, " Ranking of Heart Hospital s Using cross-efficiency and two-stage DEA," $7^{\text {th }}$ International Conference on Computer and Knowledge Engineering (ICCKE), Mashhad, 2017, pp. 217.

[3] S.M. Bucura, M. Chibeleana, A. Gligorb and M. Pacurara, Expert system for determining the level of stress before pediatric dental treatment, The 7th International Conference Interdisciplinarity in Engineering, Procedia Technology 12 (2014) 548 - 557.

[4] A. Boss, J. Hoogstraten, and B. Prahl-Andersen. On the use of personality characteristics in predicting compliance in orthodontic practice. Am J Orthod Dentofacial Orthop 2003, 123: 569-572.

[5] M. Mirmozaffari, "Presenting a Medical Expert System for Diagnosis and Treatment of Nephrolithiasis", EJMED, vol. 1, no. 1, May 2019.

[6] M. Mirmozaffari, "Developing an Expert System for Diagnosing Liver Diseases", EJERS, vol. 4, no. 3, pp. 1-5, Mar. 2019.

[7] O. A. Gashteroodkhani, M. Majidi, M. Etezadi-Amoli, "A Fuzzy-based Control Scheme for Recapturing Waste Energy in Water Pressure
Reducing Valves", IEEE Power and Energy Society General Meeting (PESGM), Portland, OR, USA, Aug 2018.

[8] M. Etribi, Expert Systems Approaches for Decision Support in Health Care, Proceeding of the Twelfth Annual Conference of the Production and Operations Management Society, 2001.

[9] S. Amin-Nejad, T. A. Gashteroodkhani, K. Basharkhah. "A Comparison of MVDR and LCMV Beamformers' Floating Point Implementations on FPGAs." Wireless Personal Communications, vol. 98, no.2, pp.1913-1929, 2018.

[10] M. Mirmozaffari, "Eco-Efficiency Evaluation in Two-Stage Network Structure: Case Study: Cement Companies". Iranian Journal of Optimization (IJO). Dec. 16, 2018. 
